\title{
GROUND ROLL COUPLING TO ATMOSPHERIC COMPRESSIONAL WAVES*
}

\author{
FRANK PRESS † AND MAURICE EWING $\dagger$
}

\begin{abstract}
A theoretical treatment of ground roll originating from air shots and hole shots is given. It is shown that coupling of ground roll to compressional waves in the atmosphere exists for both air shots and hole shots. Experimental data obtained in the field are in excellent agreement with the theoretical results; namely, that the effective coupling exists for surface waves whose phase velocity is equal to the speed of sound in air.

In regions where Rayleigh wave velocities vary with period due to layering in such a way that they are less than the speed of sound in air for short periods and excecd this value for longer periods, this coupling gives rise to a unique surface wave pattern on seismic records.

It is shown that body wave and surface wave character is almost independent of charge elevation in the range from $\circ$ (on the ground) to 30 feet.

In a reciprocal manner ground roll from hole shots was recorded with air microphones as predicted by the theory.
\end{abstract}

\section{INTRODUCTION}

Recent experimental and theoretical studies reported in the literature ${ }^{1,2}$ have shown that under certain conditions close coupling can exist between the atmosphere and surface waves of many types. When applied to Rayleigh waves, these results explain many of the characteristics of ground roll from hole shots and air shots. In particular, previously unexplained phenomena associated with air shooting methods are readily interpreted. Field conditions under which air shooting methods fail to produce significant changes in the character of ground roll can be predicted.

\section{THEORY}

An approximate theory of ground roll originating in an air shot can be given if the reaction of the Rayleigh wave on the air is neglected. In this case the air wave from the explosion can be treated simply as a pressure pulse traveling over the surface of a dispersive system. Theoretical results of $\mathrm{Lamb}^{3}$ on the effect of a traveling disturbance can be applied. He represented a traveling disturbance by a succession of infinitesimal impulses applied at equal intervals of time at points

* Presented at the St. Louis Meeting of the Society April 25, I95 I. Manuscript received by the editor February 8, $195 \mathrm{I}$.

$\dagger$ Lamont Geological Observatory (Columbia University), Palisades, New York.

$\ddagger$ Consultant to Magnolia Petroleum Company, Dallas, Texas, during performance of field work reported in this paper.

${ }^{1}$ Frank Press, A. P. Crary, Jack Oliver, Samuel Katz, "Air Coupled Flexural Waves in Floating Ice," Trans. A mer. Geophys. Union, Vol. 32 (1935), I66-1 72.

${ }^{2}$ Frank Press, Maurice Ewing, "Theory of Air Coupled Flexural Waves," in press, Journ. A ppl. Phys. (1951).

${ }^{3}$ H. Lamb, "Hydrodynamics," Cambridge University Press, 6th Edition (1932) 4113-415. 
along the path. Each impulse produces a train of waves, and superposition of all the wave trains results in reinforcement only for those waves (having a discrete frequency) whose phase velocity equals the speed of sound in air. Since energy associated with a given frequency is propagated with the corresponding group velocity, the wave system generated by an air shot consists of a train of constant frequency waves extending from the air wave arrival to the arrival time for waves traveling from the shot point with this group velocity. In a typical case the group velocity associated with the critical frequency is about half the speed of sound in air, so the surface waves begin with the air wave and have a duration about equal to the travel time. Thus, the approximate theory explains an outstanding feature of air shooting, that ground roll from an air shot is limited very often to the portion of the seismogram following the air wave, whereas, in a hole shot, ground roll covers the major part of the seismogram. If the phase velocity of Rayleigh waves in the ground never equals the speed of sound in air, resonant coupling cannot occur and ground roll from air and hole shots differ only through differences in the spectra of air shots and hole shots. Thus, in many regions having high velocity surface layers, the speed of Rayleigh waves exceeds the speed of sound in air for all periods, and the portion of a seismogram containing ground roll should not be as significantly altered by air shooting as in a region of low velocity surface material.

Further discussion requires a more extensive theory. In the derivations which follow we shall represent the weathered layer as a liquid and the subweathering rocks as a semi-infinite elastic solid. It is believed that this approximation is adequate to explain the atmospheric influence on ground roll with far less computation than for two solids for the following reasons: (I) In both cases the low frequency limit of phase velocity is the speed of Rayleigh waves in the bottom layer; (2) Setting the speed of compressional waves in the liquid layer equal to the speed of Rayleigh waves in the upper solid layer results in identical high frequency limits of phase velocity for both cases; (3) At intermediate frequencies phase and group velocity curves for the two cases are not significantly different.* It can be shown that an additional high frequency branch can exist for the two solid layer case in which phase velocity approaches the speed of compressional waves in the upper layer. This branch enters only as a higher mode and, as will be seen later, is associated with phase velocities much too high for resonant atmospheric coupling.

Consider the propagation of Rayleigh waves in a system consisting of an infinite atmosphere having density $\rho_{0}$, incompressibility $\lambda_{0}$, and sound velocity $v_{0}$ overlying a liquid layer of thickness $H$, having density $\rho_{1}$, incompressibility $\lambda_{1}$, and compressional wave velocity $v_{1}$. The liquid layer in turn overlies an elastic solid of infinite extent having density $\rho_{2}$, compressional wave velocity $\alpha_{2}$, shear

* Recent calculations of air coupled Rayleigh waves for the case of a solid surface layer give almost identical results to those obtained using a liquid surface layer. This material will be published in another paper. 
wave velocity $\beta_{2}$, and Lame's constants $\lambda_{2}, \mu_{2}$. Poisson's constant for the solid layer is taken as 0.25 and we may write:

$$
\begin{aligned}
v_{0}{ }^{2} & =\lambda_{0} / \rho_{0} \\
v_{1}{ }^{2} & =\lambda_{1} / \rho_{1} \\
\alpha_{2}{ }^{2} & =\left(\lambda_{2}+2 \mu_{2}\right) / \rho_{2} \\
\beta_{2}{ }^{2} & =\mu_{2} / \rho_{2}=\alpha_{2}{ }^{2} / 3 .
\end{aligned}
$$

A Cartesian coordinate system is chosen with the $x$-axis in the air liquid interface parallel to the direction of propagation and the $z$-axis vertically downward,

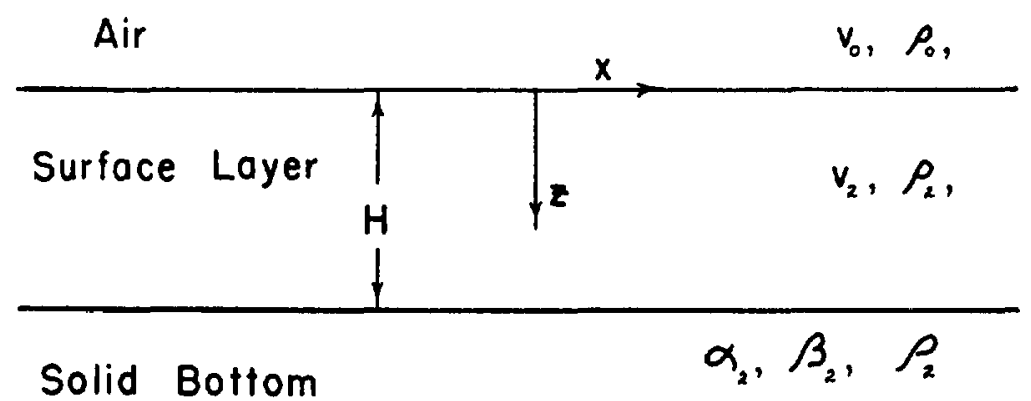

Fig. I. Assumed surface layering.

shown in Figure $\mathbf{I}$. We denote displacements parallel to $z$ and $x$ by $w$ and $u$ respectively and the subscripts $0, I, 2$ hereafter refer to air, liquid, and solid layers respectively.

Following the mathematical procedure of $\mathrm{Lamb}^{4}$ we introduce the functions $\phi(x, z, t)$ and $\psi(x, z, y)$ defined by the equations:

$$
\left.\begin{array}{rl}
u_{i} & =\partial \phi_{i} / \partial x \\
w_{i} & =\partial \phi_{i} / \partial z
\end{array}\right\} \quad i=0, \mathrm{r}
$$

Expressing the vertical stress $p_{z z}$ and the tangential stress $p_{z x}$ in terms of $\phi, \psi$ and the elastic constants we can write:

$$
\begin{aligned}
& p_{z z}=\lambda \nabla^{2} \phi+2 \mu\left(\partial^{2} \phi / \partial z^{2}-\partial^{2} \psi / \partial x \partial z\right) \\
& p_{z x}=\mu\left(\partial^{2} \psi / \partial z^{2}-\partial^{2} \psi / \partial x^{2}+2 \partial^{2} \phi / \partial x \partial z\right) .
\end{aligned}
$$

It is required that the functions $\phi$ and $\psi$ satisfy the wave equations:

"H. Lamb, "On Elastic Waves in a Plate," Proceedings of the Royal Sociely of London, A93 (I9I7), II4. 


$$
\left.\begin{array}{l}
\partial^{2} \phi_{i} / \partial t^{2}=v_{i}{ }^{2} \nabla^{2} \phi_{i} \quad i=0, \mathrm{I} \\
\partial^{2} \phi_{2} / \phi t^{2}=\alpha_{2}{ }^{2} \nabla^{2} \phi_{2} \\
\partial^{2} \psi_{2} / \partial t^{2}=\beta_{2}{ }^{2} \nabla^{2} \psi_{2}
\end{array}\right\}
$$

and the boundary conditions:

$$
\begin{aligned}
\left(p_{z z}\right)_{0} & =\left(p_{z z}\right)_{1} & \text { at } & z=0 \\
w_{0} & =w_{1} & \text { at } & z=0 \\
\left(p_{z z}\right)_{1} & =\left(p_{z z}\right)_{2} & \text { at } & z=H \\
\left(p_{z x}\right)_{2} & =0 & \text { at } & z=H \\
w_{1} & =w_{2} & \text { at } & z=H .
\end{aligned}
$$

It can readily be verified that a set of solutions of (6) and (7) are of the form:

$$
\begin{aligned}
& \phi_{0}=A e^{\eta_{0} z} e^{i(k x-\omega t)} \\
& \phi_{1}=\left[B \sin \left(\eta_{1} z\right)+C \cos \left(\eta_{1} z\right)\right] e^{i(k x-\omega t)} \\
& \phi_{2}=D e^{-\eta_{2} z} e^{i(k x-\omega t)} \\
& \psi_{2}=E e^{-\zeta_{2} z} e^{i(k x-\omega t)}
\end{aligned}
$$

where:

$$
\left.\begin{array}{l}
\eta_{0}^{2}=k^{2}-\omega^{2} / v_{0}^{2}=k^{2}\left(\mathrm{I}-\frac{c^{2}}{v_{0}^{2}}\right) \\
\eta_{1}^{2}=\omega^{2} / \nu_{1}^{2}-k^{2}=k^{2}\left(\frac{c^{2}}{v_{1}{ }^{2}}-\mathrm{I}\right) \\
\eta_{2}^{2}=k^{2}-\omega^{2} / \alpha_{2}^{2}=k^{2}\left(\mathrm{I}-\frac{c^{2}}{\alpha_{2}^{2}}\right) \\
\zeta_{2}^{2}=k^{2}-\omega^{2} / \beta_{2}^{2}=k^{2}\left(\mathrm{I}-\frac{c^{2}}{\beta_{2}^{2}}\right)
\end{array}\right\}
$$

and $k$ is the wave number related to wavelength $L$ (measured in the direction of $x)$ by $k=2 \pi / L$ and to phase velocity $c$ by

$$
c=\omega / k \text {. }
$$

In equations I $_{3}-\mathbf{I} 6, \phi_{0}, \phi_{1}, \phi_{2}, \psi_{2}$ define a system of waves propagating in the $x$ direction with phase velocity $c ; \phi_{0}, \phi_{1}, \phi_{2}$ represent compressional wave components and $\psi_{2}$ represents a distortional wave component. We will be interested in the two cases $\beta_{2} \geqq v_{0} \geqq c \geqq v_{1}$ and $\beta_{2} \geqq c \geqq v_{0} \geqq v_{1}$, which represent the conditions of a low speed surface layer. In the first case all $\eta$ 's and $\zeta$ are real, wave amplitudes decrease exponentially with distance from the surface layer and the solutions represent undamped wave guide propagation through the surface layer. 
In the second case $\eta_{0}$ is imaginary, $\phi_{0}$ is finite at infinite distances from the surface layer indicating energy radiation from the surface layer into the air.

If (13)-(16) are substituted in the boundary conditions (8)-(I2), five independent homogeneous equations in the amplitude factors $A, B, C, D, E$ are obtained. Any four of these five factors can be found in terms of the fifth, but for a solution to exist the determinant formed by the coefficients of the five factors in the homogeneous set of equations must vanish. This amounts to eliminating the wave amplitude factors between the five boundary equations. Performing this operation we obtain the frequency equation which can be written as follows after some simplification:

$$
\begin{aligned}
& \tan \left(k H \sqrt{c^{2} / v_{1}{ }^{2}-\mathrm{I}}\right) \\
& -\frac{\rho_{2}}{\rho_{1}} \frac{\beta_{2}{ }^{4}}{c^{4}} \frac{\sqrt{c^{2} / v_{1}^{2}-\mathrm{I}}}{\sqrt{\mathrm{I}-c^{2} / \alpha_{2}{ }^{2}}}\left[4 \sqrt{\mathrm{I}-\frac{c^{2}}{\alpha_{2}{ }^{2}}} \sqrt{\mathrm{I}-\frac{c^{2}}{\beta_{2}{ }^{2}}}-\left(2-\frac{c^{2}}{\beta_{2}{ }^{2}}\right)^{2}\right]
\end{aligned}
$$

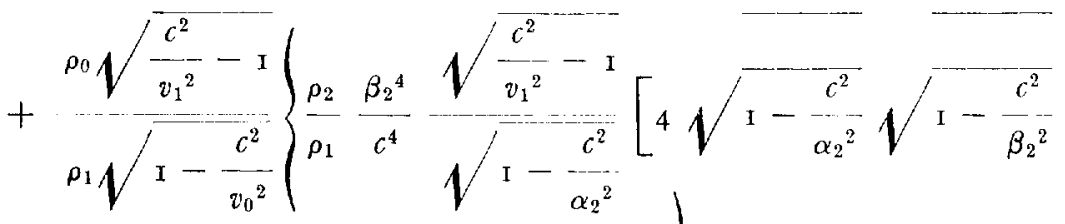

$$
\begin{aligned}
& \left.\left.-\left(2-\frac{c^{2}}{\beta_{2}^{2}}\right)^{2}\right] \tan \left(k H \sqrt{\frac{c^{2}}{v_{1}^{2}}-I}\right)+\mathbf{I}\right\}=0 .
\end{aligned}
$$

As a check on Equation (I 9 ) we see that for $\rho_{0}=0$ it reduces to the frequency equation for Rayleigh waves propagated in a liquid layer overlying a semi-infinite elastic solid, for $\rho_{0}=\rho_{1}=0$ it reduces to Rayleigh waves in the solid, and for $\rho_{2}=0$ it reduces to the frequency equation for compressional waves propagating through two liquid layers.

Equation (I9) defines an implicit relationship between frequency $f=c k / 2 \pi$ and phase velocity $c$ with the elastic constants of the system as parameters. Since $\rho_{0} / \rho_{1}$ is of order of 0.001 , the third term in the equation which gives the effect of the atmosphere is negligible except in the region $c$ close to $v_{0}$. For $\beta_{2} \geqq v_{0} \geqq c \geqq v_{1}$ real values of $k$ and $c$ satisfying (I9) can be found. For $c$ real and $\beta_{2} \geqq c>v_{0}$ the first and second terms of ( 19 ) are real, the third term is imaginary, hence their sum can never vanish. Actually it can be shown that for this range of values of $c$ the effect of the third term is to add a very small imaginary component to the real values of $c$ determined by neglecting the third term, except, of course, when $c$ is close to $v_{0}$ and the imaginary component becomes large. Complex values of phase velocity correspond to radiation of energy into the atmosphere, the amount of radiation increasing with the magnitude of the imaginary component.

Equation (Ig) was used to compute $k$ as a function of $c$ for the case 
$\rho_{2} / \rho_{1}=$ I.I $, \quad \rho_{0} / \rho_{1}=0.00 \mathrm{I}, \quad \alpha_{2}=\sqrt{3} \beta_{2}, \quad \beta_{2}=2.5 v_{1}, \quad v_{0}=$ I. $4 v_{1}$. which were chosen as the most typical values for weathered layers. Group velocity was computed by numerical differentiation using the equation:

$$
U=c+k \frac{d c}{d k}
$$

Results of these computations are presented in dimensionless form in Figure 2, where $c / v_{1}$ and $U / v_{1}$ are plotted against $k H$ for the lowest mode of vibration. The dimensionless parameter $k H$ is proportional to frequency through the equa-

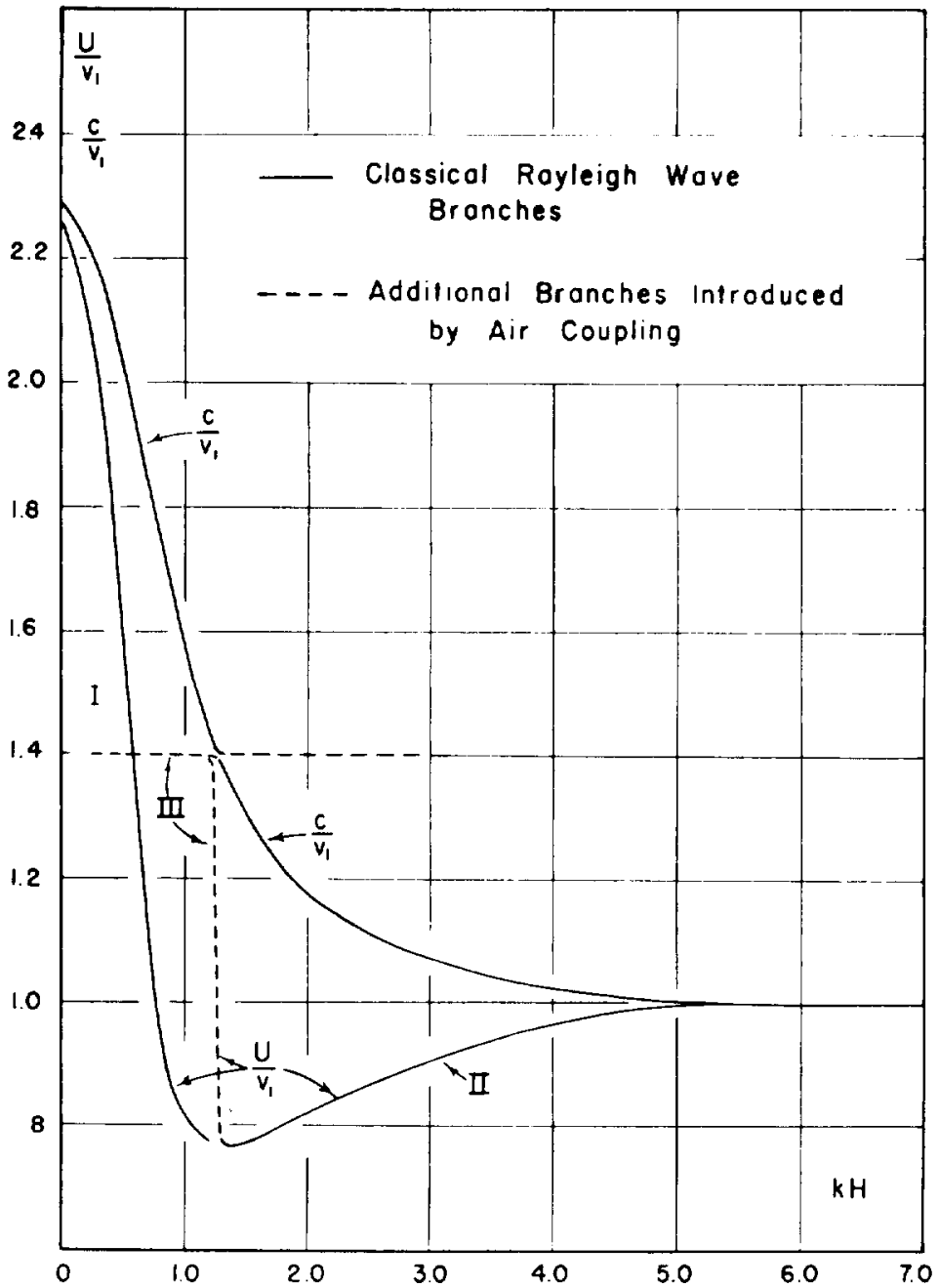

FIG. 2. Phase and group velocity of air coupled Rayleigh waves. 
tion $f=\left(c / v_{1}\right) k H / 2 \pi\left(H / v_{1}\right)$. The heavy lines in Figure 2 show where atmosphere influence is negligible and the dashed lines are new branches introduced by air coupling.

It is well known that in a dispersive medium such as the one under consideration, the sequence of waves arriving at a given point can be obtained from a group velocity curve such as that presented in Figure 2. For discussion of the amplitudes of waves corresponding to the different branches of the group velocity curve, one must derive the excitation functions for hole shots and air shots. These functions relate wave amplitudes to the position of the source and the frequency spectrum of the pulse which it emits. (See for example, Pekeris ${ }^{5}$ or Press and Ewing ${ }^{6}$ ) Although the mathematical procedure is straightforward the computations are very long. Thus, the group velocity curve of Figure 2 can be used to determine the arrival time and frequency of ground roll for sources in any part of the system (e.g., air shots or hole shots) as recorded by pickups located in any part of the system (e.g., geophones or air microphones) but the relative amplitudes of waves for different source and pickup locations cannot be given. Existing calculations for air coupling to another type of surface wave (reference 2) can be used, however, to infer something about relative amplitudes.

We have divided the group velocity curve of Figure 2 into branches I, II, and III, each of which represents a different train of waves. Branch I accounts for the dispersive train of Rayleigh waves usually associated with ground roll. Waves corresponding to this branch first appear as low frequency arrivals $(k H \approx 0)$ travelling with the speed of Rayleigh waves in the bottom layer $\left(U \approx 2.3 v_{1}=.92 \beta_{2}\right)$. Subsequent arrivals show a gradual increase in frequency since in Branch $\mathbf{I} k H$ increases as the group velocity decreases. The wave train continues to arrive with increasing frequency until a time corresponding to propagation at a minimum value of group velocity $U \approx{ }_{7} 8 v_{1}$. From the analogous theory referred to above we would expect waves propagated according to Branch I to predominate for a hole shot recorded by a surface geophone. Note that the lower group velocity waves propagated according to Branch I have phase velocities approaching the speed of sound in air from the side $c>v_{0}$. These waves are therefore highly attenuated due to the large imaginary component of phase velocity. The fact that the minimum value of group velocity has a corresponding phase velocity close to $v_{0}$ is fortuitous in that it depends on the values of $\beta_{2} / v_{1}$ and $v_{0} / v_{1}$. Our values for these constants, however, seem most representative of field conditions encountered in most of the experiments.

Branch II represents a train of higher frequency waves controlled essentially by propagation within the surface layer. Waves propagated according to this branch have been observed at sea where the surface layer is water, but have

${ }^{5}$ C. L. Pekeris, "Theory of Propagation of Explosive Sounds in Shallow Water," in Propagation of Sound in the Ocean, Mem. 27, Geological Society of America (1948).

${ }^{6}$ Frank Press and Maurice Ewing, "Propagation of Explosive Sound in a Liquid Layer Overlying a Semi-Infinite Elastic Solid," Geophysics, Vol. XV, No. 3 (1950), 426-446. 
never been observed on land. It is probable that water, having excellent elastic properties, transmits these high frequency waves freely, whereas, the poorly elastic surface weathered layer absorbs and scatters most of the higher frequency energy.

Branch III results from coupling of the ground roll to atmospheric compressional waves. Waves propagated according to this branch begin abruptly with the arrival of the air wave $\left(U=\mathbf{I} .4 v_{1}, \circ \leqq k H \leqq \mathrm{I} .2\right)$ and are followed by a train of constant frequency waves $(k H \approx \mathrm{I} .25)$, having constant phase velocity $c=v_{0}$ and lasting until the time corresponding to propagation at the value of group velocity $U=.78 v_{1}$ found when $k H \approx \mathrm{r} .25$. Note that the single frequency of the waves following the air pulse is identical to that of the ground roll whose phase velocity equals the speed of sound in air-in agreement with the approximate theory. From the results of the analogous theory of flexural waves (reference 2), we would expect waves propagated according to Branch III to predominate for air shots recorded by surface geophones and reciprocally for hole shots recorded by air microphones. From Equations $\left(\mathrm{I}_{3}\right)$ and $\left(\mathrm{I}_{7}\right)$, we note that the amplitude of the air motion decreases with height above the surface as $e^{-k z \sqrt{1-c^{2} / v_{0}^{2}}}$. It can be shown in a reciprocal manner that for shots in the air, all amplitudes decrease as $e^{-k d \sqrt{1-c^{2} / v_{0}^{2}}}$, where $d$ is the height of the shot point above the surface. Since $c=v_{0}$ for the constant frequency wave train propagated according to Branch III, it follows that the amplitude of these waves is independent of the height of the air charge above the surface, as long as $d / L$ is finite and $d \ll$ shot-detector distance. We may summarize our theoretical results as follows:

I. For a hole shot in a layered medium such as that postulated a geophone records ground roll as an orderly sequence of dispersive Rayleigh waves.

2. For a hole shot an air microphone records a constant frequency train of waves following the abrupt arrival transmitted with the speed of sound in air.

3. Ground roll from an air shot recorded by a geophone predominates after the arrival of the direct air wave, and then only as a train of waves having constant frequency and phase velocity. The character of the seismogram after the arrival of the air wave is independent of the height of the charge above the surface.

4. The different wave trains described all arise from a single source. Multiple charges can be represented by superposition of the wave trains originating in each source to the extent that non-linear effects are negligible.

5. Dispersive Rayleigh waves can be produced by propagation in a medium having a gradual increase in shear velocity with depth. Although the exact theory has not been worked out one would expect air coupling results analogous to the layer theory.

6. In areas where a high speed surface layer occurs such that the Rayleigh wave velocity in the surface layer exceeds the speed of sound in air, coupling of ground roll to the air cannot exist. One would therefore expect only minor differences in ground roll from air shots and hole shots-these originating in differences in the source spectra for the two types of shots. 


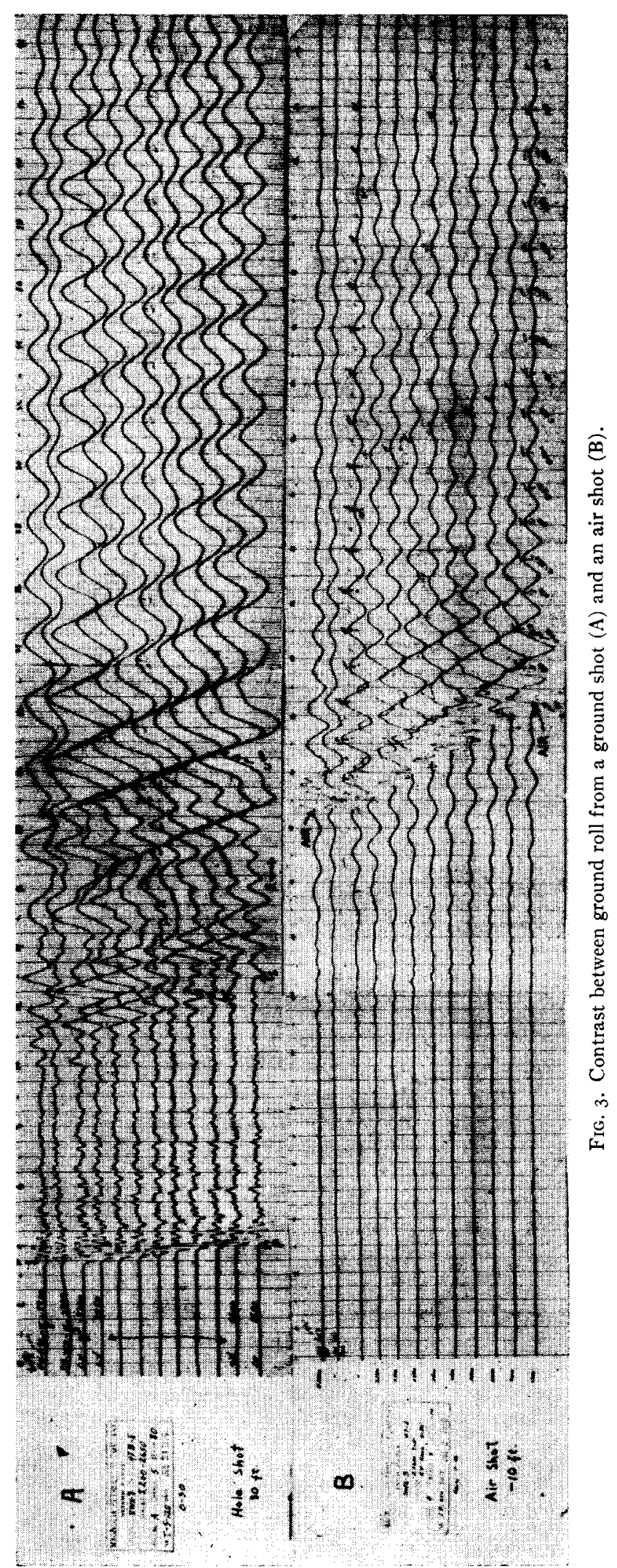




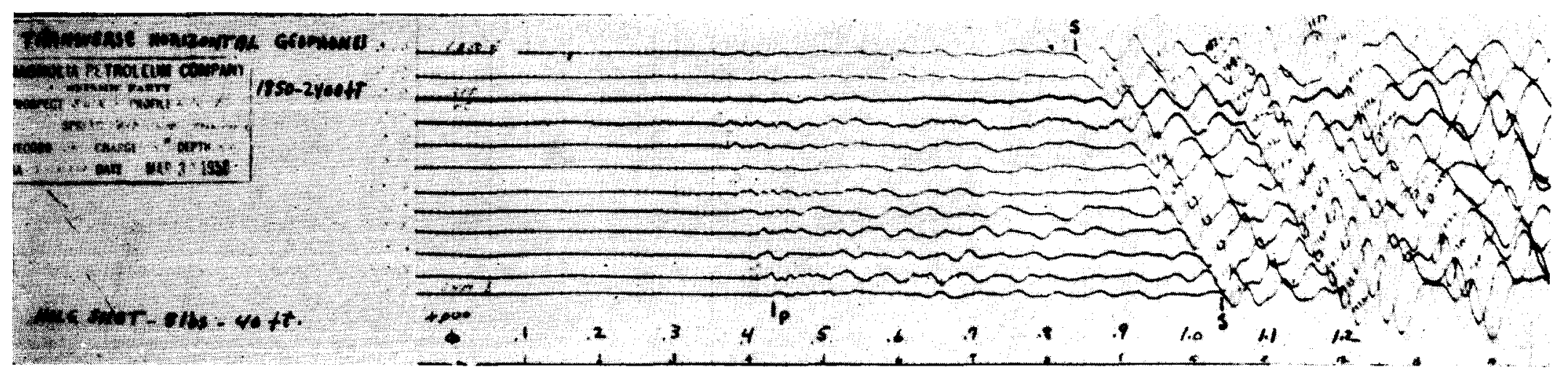

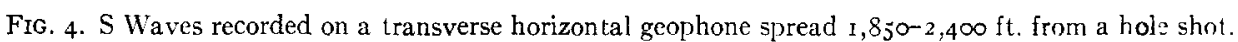




\section{EXPERIMENTAL RESULTS}

In the course of a series of field experiments on surface waves, sufficient data were obtained to provide striking verification of the theoretical results. In general it was found that agreement was best in areas where ground roll from a hole shot appeared as an orderly sequence of dispersive Rayleigh waves.

In Figures 3, 6, 7, single 5-pound charges where detonated in holes, on the ground and in the air as specified on each record. Spread distance was $2,200-2,65^{\circ}$

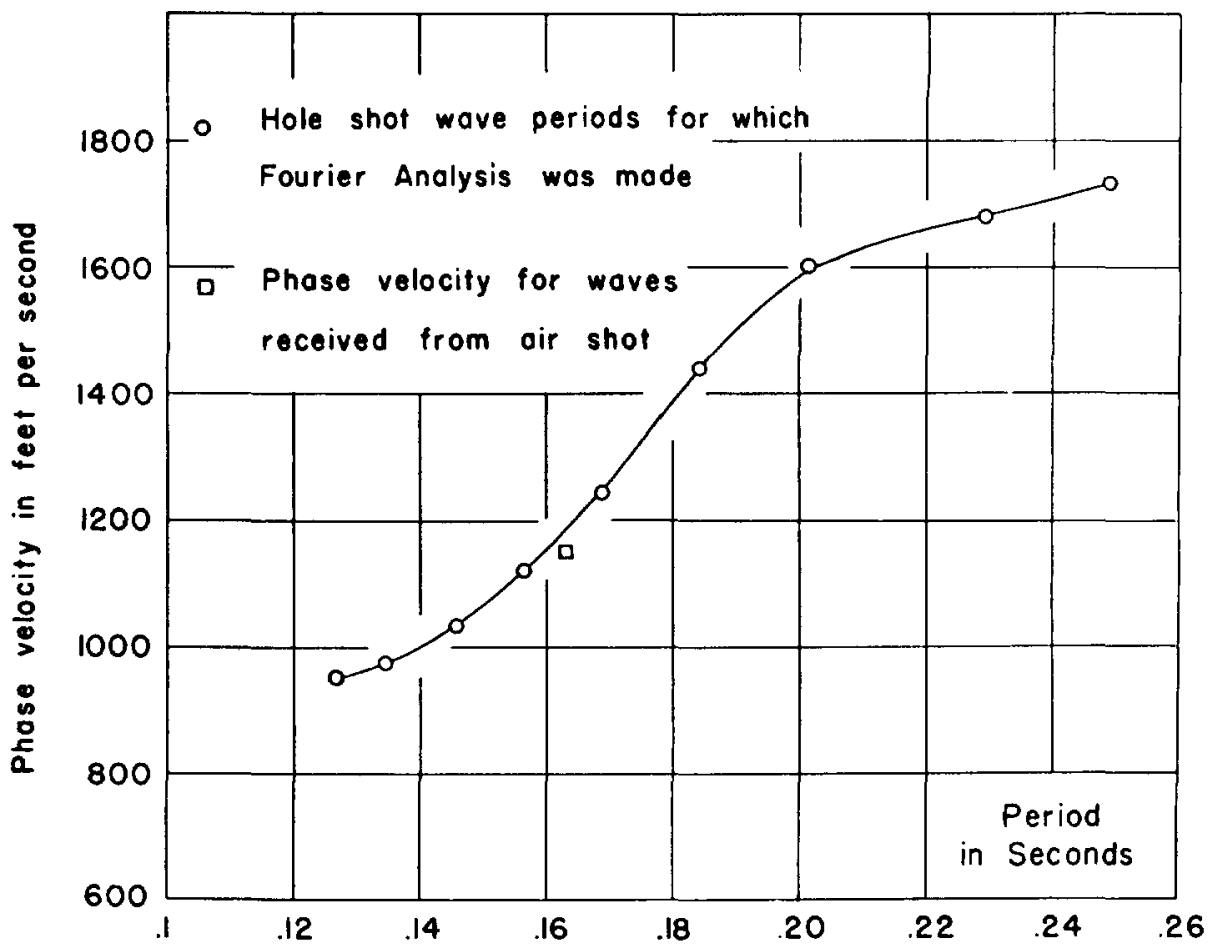

FIG. 5. Phase velocity curves for hole shot and air shot by Fourier analysis of records at 800 feet and 1,200 feet.

feet and the first three traces are reception at 2,200 feet from (I) radial horizontal; (2) a trace (spurious due to a recording error) originally intended to be transverse horizontal; and (3) vertical geophone. Succeeding traces are vertical geophones located at 50 -foot intervals.

Wide band amplifiers passing $5^{-220} \mathrm{cps}$ were used with integrating circuits to give ground displacement.

Figure 3 contrasts the ground roll from a hole shot (A) and an air shot (B) at the same shot point. Refracted compressional waves $(P)$ and waves tentatively identified as shear $(S)$ are followed by dispersive Rayleigh waves $(R)$ in the hole shot record. 


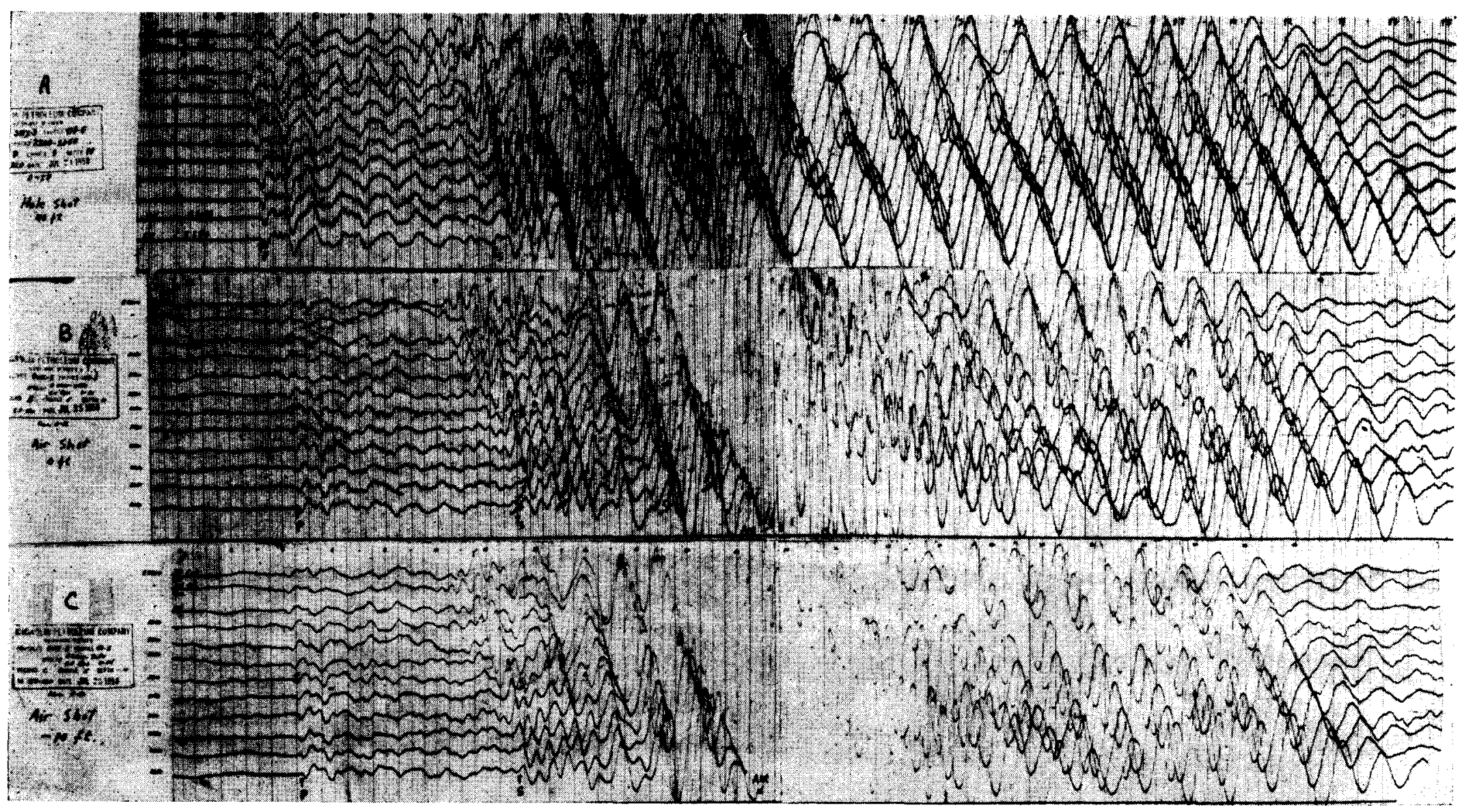

Fic. 7. Comparison of body waves and surface waves from hole shot (A), single air shots (B and C). 
The main evidence in favor of identifying the second group as shear waves is ( $\mathrm{I}$ ) the large transverse component in these waves as shown in Figure 4 which consists of a transverse horizontal spread I,850-2,400 feet; (2) the agreement of velocity with borehole determinations. A difficulty with this interpretation is the improper phase relationship between the vertical and radial horizontal geophone at 2,200 feet as shown in Figures $3 \mathrm{~A},{ }_{7} \mathrm{~B}$, and ${ }_{7} \mathrm{C}$. Additional study of these waves is needed.

The Rayleigh waves are identified on the basis of their retrograde elliptical motion, their characteristic dispersion, and their low velocity. In many cycles the axis of the elliptical orbit is inclined with the vertical, a phenomenon also observed by Howell. ${ }^{7}$

Ground roll on the air shot record consists of relatively constant frequency Rayleigh waves immediately following the direct air wave. The phase velocity of these waves remains reasonably close to the speed of sound in air for about 6 cycles, and thereafter increases erratically by about 30 per cent. With the use of a Fourier analyzer phase velocity was obtained as a function of frequency for the dispersive Rayleigh waves. These results are shown in Figure 5 for records obtained at 800 feet and $I, 200$ feet. It was found that the frequency of waves whose phase velocity equalled the speed of sound in air agreed with the frequency of the waves following the air pulse on the air shot records. The characteristics of ground roll for air shots and hole shots thus bear out our theoretical results.

Figure 6 shows the effect of elevation of charge on the character of ground roll for an air shot, all other factors including amplifier gain remaining the same. It is seen that record character remains unchanged for shot heights ranging from - -30 feet, as predicted by the theoretical results.

Figures ${ }_{7} \mathrm{~B}$ and ${ }_{7} \mathrm{C}$ are records of air shots detonated at elevation of 0 feet and to feet and recorded at higher gains than the previous air shot records. The constant frequency Rayleigh waves following the air wave are almost off scale. The predominant disturbances preceding the air waves are $P$ and $S($ ?) type body waves. Note that the body waves are almost identical for the two shots suggesting that energy transfer into body waves is not critically dependent on the elevation of an air shot. Comparison with the hole shot in Figure $7 \mathrm{~A}$ shows that the character of the body waves from the hole shots and the air shots are similar. From records obtained with different spreads here and at other locations we conclude that the ratio of surface waves to body waves after the time necessary for the air wave to arrive is many times greater for an air shot than for a hole shot of identical charge size at the same location.

On many occasions microphones several feet above the ground recorded constant frequency air coupled waves from hole shots, providing a convincing verification of the theoretical results given in the previous section.

${ }^{7}$ B. F. Howell, Jr., "Ground Vibrations Near Explosions," Bull. S.S.A., Vol. 39 (1949), 285-310. 


\section{ACKNOOWLEDGMENTS}

We are grateful to the Magnolia Petroleum Company for permission to publish much of the material included in this paper. We wish to acknowledge the contributions of Dr. M. B. Dobrin, Dr. J. E. White, and Messrs. H. H. Frost, R. L. Sengbush, P. L. Lawrence, A. G. Hubby, W. G. Hicks, and J. H. Henkel for aid in performing and interpreting the experimental work.

(Lamont Geological Observatory Contribution No. 32) 